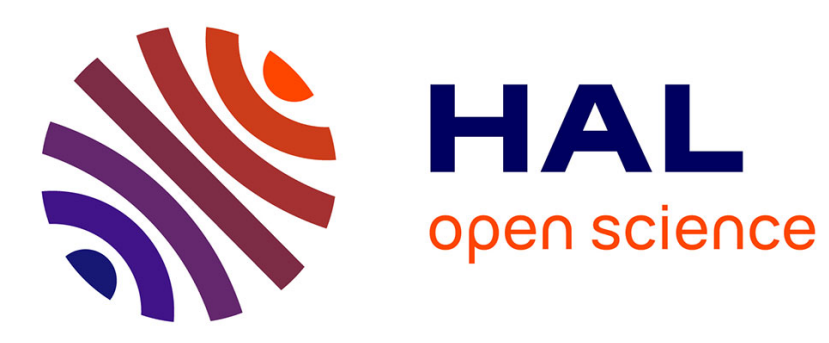

\title{
Identification of the hygrothermal properties of a building envelope material by the Covariance Matrix Adaptation evolution strategy
}

Simon Rouchier, Monika Woloszyn, Yannick Kedowide, Timea Béjat

\section{- To cite this version:}

Simon Rouchier, Monika Woloszyn, Yannick Kedowide, Timea Béjat. Identification of the hygrothermal properties of a building envelope material by the Covariance Matrix Adaptation evolution strategy. Journal of Building Performance Simulation, 2015, 10.1080/19401493.2014.996608 . hal-01288774

\section{HAL Id: hal-01288774 \\ https://hal.science/hal-01288774}

Submitted on 15 Mar 2016

HAL is a multi-disciplinary open access archive for the deposit and dissemination of scientific research documents, whether they are published or not. The documents may come from teaching and research institutions in France or abroad, or from public or private research centers.
L'archive ouverte pluridisciplinaire HAL, est destinée au dépôt et à la diffusion de documents scientifiques de niveau recherche, publiés ou non, émanant des établissements d'enseignement et de recherche français ou étrangers, des laboratoires publics ou privés. 


\title{
Identification of the hygrothermal properties of a building envelope material by the Covariance Matrix Adaptation evolution strategy
}

\author{
Simon Rouchier $^{a * *}{ }^{*}$ Monika Woloszyn ${ }^{a}$, Yannick Kedowide ${ }^{a}$ and Timea Béjat ${ }^{b}$ \\ ${ }^{a}$ LOCIE, CNRS-UMR5271, Université de Savoie; Campus Scientifique, Savoie Technolac, 73376 \\ Le Bourget-du-Lac Cedex, France \\ ${ }^{b}$ Univ. Grenoble Alpes, INES, F-73375 Le Bourget du Lac, France \\ CEA, LITEN, Department of Solar Technologies, F-73375 Le Bourget du Lac, France
}

Postprint: Rouchier S, Woloszyn M, Kedowide Y, Bejat T 2013. Identification of the hygrothermal properties of a building envelope material by the Covariance Matrix Adaptation evolution strategy, Journal of Building Performance Simulation DOI: 10.1080/19401493.2014.996608

\begin{abstract}
This paper proposes the application of the Covariance Matrix Adaptation (CMA) evolution strategy for the identification of building envelope materials hygrothermal properties. All material properties are estimated on the basis of local temperature and relative humidity measurements, by solving the inverse heat and moisture transfer problem. The applicability of the identification procedure is demonstrated in two stages: first, a numerical benchmark is developed and used as to show the potential identification accuracy, justify the choice for a Tikhonov regularisation term in the fitness evaluation, and propose a method for its appropriate tuning. Then, the procedure is applied on the basis of experimental measurements from an instrumented test cell, and compared to the experimental characterisation of the observed material. Results show that an accurate estimation of all hygrothermal properties of a building material is feasible, if the objective function of the inverse problem is carefully defined.
\end{abstract}

Keywords identification; evolutionary algorithm; CMA; heat and moisture transfer; modelling

\section{Introduction}

In the general scope of building energy retrofitting, the diagnosis of the structural and transfer properties of the envelope prior to its renovation is essential as to identify potential sources for improvement and propose costefficient solutions. As we want this diagnosis to be as comprehensive as possible, there is great interest in making techniques for in-situ characterisation available, possibly in a non intrusive way. As moisture is one of the predominant causes for damage in building materials, the local identification of the actual moisture transfer properties of building materials can be of great importance for a sustainable retrofitting.

The procedure for such a characterisation is to solve the inverse problem of identifying model parameters from dynamic measurements. The term of model calibration is sometimes used. It resembles an optimisation process,

\footnotetext{
**Corresponding author. Email: simon.rouchier@univ-savoie.fr
} 
in which the objective is to minimise a residual between measurements and predictions. Thus, most methods used to this aim are inspired by the field of optimisation, already largely applied to building physics [7].

The first category of techniques is the set of gradient-based, deterministic optimisers for non-linear least square problems [6]: the Gauss-Newton and Levenberg-Marquardt algorithms are the most common among this class of techniques. These methods use the Jacobian or the Hessian matrix of the residuals and are widely used for solving the inverse heat transfer problem [23]. If the gradient of the functional to minimise is not available, as is generally the case when this objective function is the result of a set of partial differential equations solved by the finite element method, the adjoint state method can be used to generate an approximation of the Jacobian matrix. Recent work has shown the applicability of this procedure to the identification of building thermal parameters $[5,25]$.

Stochastic methods constitute a second class of optimisation techniques adaptable to inverse problems. A first category may be mentioned, namely the Bayesian inference method [4]. This technique does not originate from the field of optimisation and is clearly distinct from the paradigm of least square residual minimisation. It describes sought parameters as probability density functions and returns not only point and spread estimates of the likely solutions, but also a complete description of their uncertainty, conditioned by eventual measurement noise and inaccuracy. Many applications of the Bayesian framework to the inverse heat transfer problem can be mentioned $[18,31,35]$. Its application to matters of building energy, while still marginal, seems to gain interest $[39,16,3]$.

Metaheuristic evolutionary algorithms constitute a separate category of stochastic inverse methods. Their flexibility and the possibility of multi-objective search have made them a popular choice for building design optimisation $[7,22,9,21,26]$ and inverse heat transfer problems [27, 10]. Their computational cost, higher than that of gradient descent techniques, can be reduced by the use of surrogate models [33] or model reduction. Some examples of genetic algorithms applied to the fitting of resistances and capacitances in building nodal models are also available [20, 36, 38].

The present work proposes the application of the Covariance Matrix Adaptation evolution strategy (CMA-ES) [13] to the identification of hygrothermal transfer and storage properties of walls. Evolution strategies are a subclass of evolutionary algorithms whose endogenous parameters (such as population size or mutation strength) are updated during the evolution [2], and the CMA-ES lies on the adaptation of the mutation strength as to guide the search towards favorable solutions. Our study investigates whether this method, supported by a sufficient amount of measurements, may provide a complete hygrothermal characterisation of building materials: heat conductivity and capacity, water vapour permeability and sorption isotherm, all of which are likely to be influenced by the local temperature and moisture content.

The motivation of this work is twofold. The first target, which has already been stated, is the ability to characterise building materials in place where sample extraction is not possible. Learning material properties should then be done in the least intrusive manner possible: this will be discussed upon analysing the results of the work. The second motivation is to propose new methods for testing materials in lab, where traditional characterisation methods are restrictive and time-consuming.

Sec. 2 introduces the parametrisation of the physical model and the related inverse problem. The need for some form of regularisation of the inverse problem is described. Then, a short description of the principle of CMA-ES is provided, along with motivations for its choice over other numerical methods. Sec. 3 shows how a numerical benchmark was developed and used to validate the choice of the so-called L-curve method for a proper regularisation of the problem. The confrontation of the procedure with experimental results is then shown in Sec. 4. Conclusions of the study are finally drawn in Sec. 5 .

\section{Theory and problem formulation}

\subsection{Forward and inverse problems}

The physical problem is summarized on the upper part of Fig. 1: a single-layered wall of an unknown material separates two ambiances, either controlled or uncontrolled. One-dimensional coupled heat, air and moisture (HAM) transfer through this wall is monitored by sensors (thermocouples, capacitive humidity sensors, and/or fluxme- 


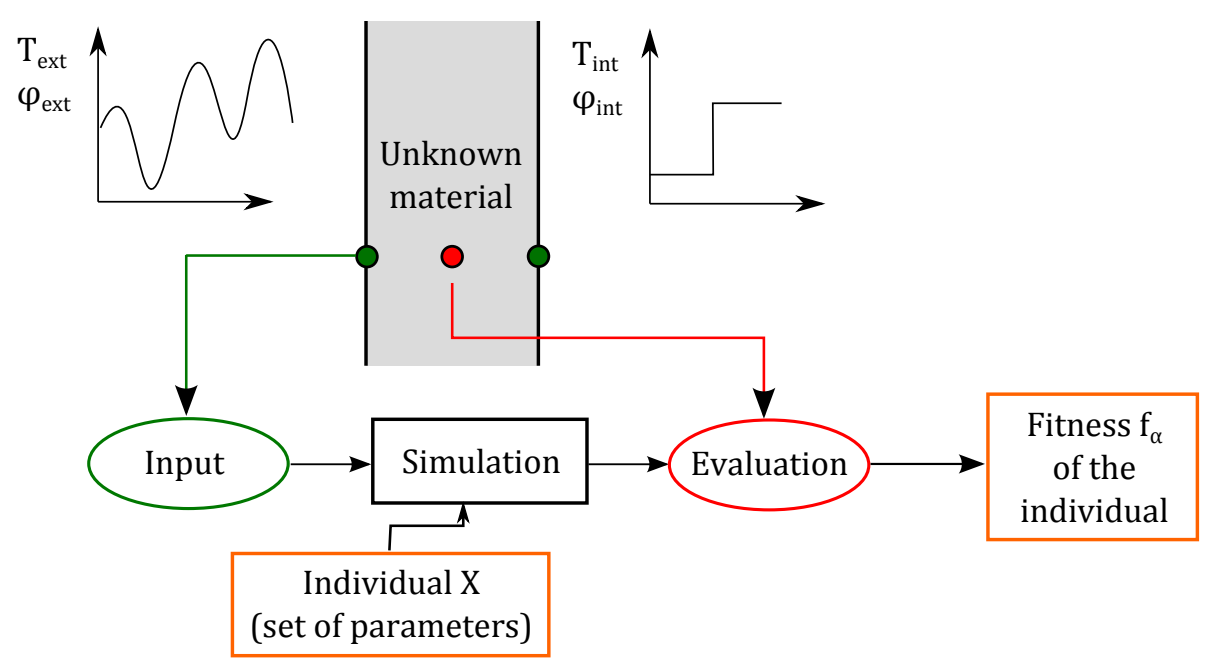

Figure 1: Outline of the material identification problem

ters) placed inside it and on its surface. Surface sensors provide boundary conditions for the HAM simulation, while a number of sensors provide reference measurements to be used for the evaluation of a candidate material.

The physical model for the simulation is the system of partial differential equations for coupled heat and moisture transfer, written here with the temperature $T$ and the vapour pressure $p_{\nu}$ as driving potentials. These conservation equations follow the notations and hypotheses usually made in building physics applications [11, 17, 30]. For the sake of simplification, liquid transfer is not considered.

$$
\begin{gathered}
\frac{\partial w}{\partial t}-\nabla \cdot\left[\delta_{p} \nabla p_{\nu}\right]=0 \\
\left(c_{p} \rho+c_{p, l} w\right) \frac{\partial T}{\partial t}+\left(c_{p, l} T\right) \frac{\partial w}{\partial t}-\nabla \cdot\left[k \nabla T+L_{\nu} \delta_{p} \nabla p_{\nu}\right]=0
\end{gathered}
$$

where $w\left[\mathrm{~kg} / \mathrm{m}^{3}\right]$ is the moisture content of the porous material, $\delta_{p}$ [s] is its vapour permeability, $c_{p} \rho\left[\mathrm{J} /\left(\mathrm{m}^{3} . \mathrm{K}\right)\right]$ its volumetric thermal capacity and $k[\mathrm{~W} /(\mathrm{m} . \mathrm{K})]$ its thermal conductivity. $c_{p, l}$ and $L_{v}$ are the specific heat and latent heat of evaporation of water. The relationship between the relative humidity $\phi$ and the moisture content within the material $w$ is given by the sorption isotherm, or moisture retention curve.

All material properties are assembled into a vector of unknowns $X \in \mathbb{R}^{n}$, where $n$ is the dimension of the search space, i.e. the total number of sought real-valued parameters. This dimension depends on the number of unknown properties and their parametrization: time-varying quantities, or for instance a temperature-dependent thermal conductivity, are approached by a set of elementary functions in order to reduce them to a finite number of parameters. In this work, none of the material properties are assumed to be previously known, and most are functions of either the temperature or the moisture content, as summarized in Tab. 1.

- The thermal conductivity $k$ is the addition of a constant value $k_{0}$ and of (independent) linear dependencies on the moisture content $w$ and temperature $T$, respectively noted $k_{w}$ and $k_{t}$. In Tab. $1, \rho_{l}$ refers to the density of liquid water.

- The vapour permeability is a linear function of the relative humidity $\phi$, and defined by an interpolation between two values that are measurable by dry cup and wet cup experiments (respectively $\phi=25 \%$ and $75 \%)$.

- The sorption isotherm is computed from its derivative $\xi=\partial w / \partial \phi$, which is defined by a second-degree polynomial interpolation between three reference points. This means that the sorption isotherm is represented by a third-degree polynomial. 
Table 1: Formulations of material properties

\begin{tabular}{|llc|}
\hline Variable & Formulation & Unknowns \\
\hline Thermal capacity & Constant & 1 \\
$c_{p} \rho\left[\mathrm{J} /\left(\mathrm{m}^{3} . \mathrm{K}\right)\right]$ & & 3 \\
\hline Thermal conductivity & Linear dependency on $T$ and $w$ & \\
$k[\mathrm{~W} /(\mathrm{m} . \mathrm{K})]$ & $k_{0}+k_{m} \frac{w}{\rho_{l}}+k_{t} T$ & 2 \\
\hline Vapour permeability & Linear interpolation between two values & \\
$\delta_{p}[\mathrm{~s}]$ & {$\left[\delta_{p, 25 \%}, \delta_{p, 75 \%}\right]$} & 3 \\
\hline Sorption isotherm & Derivative $\xi$ given by a second-degree polynomial & \\
$w\left[\mathrm{~kg} / \mathrm{m}^{3}\right]$ & {$\left[\xi_{25 \%}, \xi_{50 \%}, \xi_{75 \%}\right]$} & \\
\hline
\end{tabular}

These choices for the formulations of material properties result in an unknown vector belonging to a search space of dimension $n=9$ :

$$
X=\left\{c_{p} \rho, k_{0}, k_{m}, k_{t}, \delta_{p, 25 \%}, \delta_{p, 75 \%}, \xi_{25 \%}, \xi_{50 \%}, \xi_{75 \%}\right\}
$$

There is some flexibility in the dimension of $X$ when setting the hypotheses of the inverse problem formulation. A smaller search space usually facilitates the identification procedure. Reducing the dimensionality of the problem can, for instance, be justified by a preliminary sensitivity analysis which would designate some variables as little influential on the solution of the forward problem. Inversely, one may want to draw some constitutive law of the studied material with a higher resolution. The sorption isotherm, for instance, may be approximated by a larger set of reference points, which would be similar to a more precise experimental characterisation with additional measurement points. A fair compromise is necessary, as to avoid a raw approximation of the material properties while allowing the algorithm to run efficiently.

The numerical implementation of Eq. 1 follows the Finite-Element Method, in the simulation code hamopy ${ }^{1}$ developed in the Python language. The Galerkin weighted-residual method was used for the spatial discretisation over a one-dimensional mesh of quadratic elements. The temporal discretisation follows the first-order implicit scheme. As the discretised system is non-linear, the solution is approached iteratively at each time step, and a Newton-Raphson iterative scheme was used as to accelerate convergence. More details on the numerical implementation are available in [28].

The form of the mathematical model described by Eq. 1, along with its numerical implementation described above, are fixed assumptions. The entire identification procedure of this work does not question the abitity of the model to recreate the physical reality. The algorithm however calculates the parameters with which a given model will have the best fit with measurements, and will be most able to recreate the reality for future tests.

The solution of the forward problem is the fitness function $f$ of $X$ given a pre-defined value of the regularisation parameter $\alpha$ :

$$
f_{\alpha}(X)=\left\|Y-Y_{m}\right\|^{2}+\alpha\left\|X-X_{p}\right\|^{2}
$$

where $Y(X)$ is the vector of outputs of the simulation code, given the set of input parameters $X . Y_{m}$ is the vector of experimental measurements and $X_{p}$ is the prior, which is an initial guess of the parameter vector $X$. The second term of Eq. 3 comes from the principle of Tikhonov regularisation [32]: it is a form of constraint added to the fitness function in order to filter physically aberrant solutions to the inverse problem. The motivation behind regularisation, along with guidelines for its tuning, is addressed in Sec. 2.2

Let us note $m$, the size of the vectors $Y$ and $Y_{m}$, which is the number of measurements, i.e. the product of the number of sensors by their sampling time step. The inequality $n<m$ is clearly a necessary condition for the feasibility of the identification, but it is not a sufficient condition. The most frequent way to evaluate the fitness of a candidate vector $X$ is to rate the approval of its simulation result $Y(X)$ with the experimental measurements by a least square difference. Hence, the first term of Eq. 3 develops as:

$$
\left\|Y-Y_{m}\right\|^{2}=w_{T}\left\|T-T_{m}\right\|^{2}+w_{\phi}\left\|\phi-\phi_{m}\right\|^{2}+w_{Q}\left\|Q-Q_{m}\right\|^{2}
$$

\footnotetext{
${ }^{1}$ hamopy: Heat, Air and Moisture transfer in Python https://code.google.com/p/hamopy/
} 
where $T, \phi$ and $Q$ are the vectors of temperature, relative humidity and heat flux calculated at the same locations, and with the same temporal discretisation, as the experimental measurements are given (here noted with the index $m$ ). This term quantifies the agreement between the model and the physical reality. It is a weighted sum of the least square difference of each measured quantity, where $w_{T}, w_{\phi}$ and $w_{Q}$ are weighting coefficients. Following standard practice, confirmed by a preliminary work conducted on a simple numerical benchmark [29], these weights are set to the inverse square of the measurement uncertainties for each quantity. Noting for instance $\Delta T$ the uncertainty on the temperature measurement provided by a thermocouple, then $w_{T}$ becomes:

$$
w_{T}=\left[\frac{1}{\Delta T}\right]^{2}
$$

This formulation assigns a low weight to inaccurate sensors as to compensate for the higher mismatch between its measurements as the predictions. Another advantage of this choice for the weighting coefficients is that all terms of Eq. 4 lay within the same order of magnitude. Supposing $N_{s}$ is the number of temperature sensors and $N_{d}$ the number of data samples, the first term of Eq. 4 becomes:

$$
w_{T}\left\|T-T_{m}\right\|^{2}=\frac{1}{N_{s}} \sum_{i=1}^{N_{s}}\left[\left(\frac{1}{\Delta T^{i}}\right)^{2} \frac{1}{N_{d}} \sum_{k=1}^{N_{d}}\left(T^{i, j}-T_{m}^{i, j}\right)^{2}\right]
$$

where $T^{i, j}$ and $T_{m}^{i, j}$ are respectively the $j$-th calculated and measured values of the temperature at the location of the $i$-th sensor. Note that the simulation code may follow finer spatial and time discretisations, but only values corresponding to measurements are used in the fitness evaluation.

Given a specific value of the regularisation parameter $\alpha$, the measurement data $Y_{m}$ and the prior $X_{p}$, the forward problem is well-posed and its solution $f_{\alpha}(X)$ is fully specified as long as the computational scheme is stable and the solution is not mesh-sensitive. The inverse problem is to find an individual $\widehat{X}$ that minimises the fitness function, given a set of measurements $Y_{m}$ :

$$
\widehat{X}=\arg \min \left\{f_{\alpha}(X)\right\}=\arg \min \left\{\left\|Y(X)-Y_{m}\right\|^{2}+\alpha\left\|X-X_{p}\right\|^{2}, X \in \mathbb{R}^{n}\right\}
$$

Thus, the target is to find the individual $\widehat{X}$ that yields a simulation result $Y$ closest to the measurements, within reasonable range from a prior knowledge of the expected parameter values. The importance of introducing such a prior knowledge in the fitness function is addressed in the following section.

\subsection{Regularisation}

It is tempting to formulate the fitness function (Eq. 3) with only the square difference between measurements and simulation results: we may instinctively admit that the set parameters resulting in the closest fit to the experimental data necessarily depict the real material properties. This ideal case is however never met in reality.

The identification procedure is a series of experimental and numerical steps along which lay several sources of errors [24]:

- the forward problem is an approximation of the modelled physical process, with a given spatial discretisation

- a hypothesis on the model may be excessively simplifying or the parametrization of a function may be wrong

- the intrusiveness of a sensor may be overlooked

- measurements are affected by noise and depend on sensor calibration, etc.

These errors, most of which cannot be quantified, add up to an estimation error on the material properties. Should the sensitivity matrix of the inverse problem be badly conditioned, a global optimum to Eq. 7 may be found with unrealistic physical values for the material properties [19]. The estimation error may rise quickly due to even a moderate measurement noise. Regularisation aims at reducing the effect of data inaccuracy on the identification. 
The first possible approach for regularisation is to reduce the degrees of freedom of the problem by restricting the search to a set of admissible solutions. It is the principle of the truncated singular value decomposition technique [14] and the future information method [1]. The second approach, known as Tikhonov regularisation [32], is another way to introduce a constraint by penalizing the fitness value of unrealistic solutions.

The fitness function $f$ (Eq. 3) is modified after this principle. A quadratic term is introduced to $f$, adding a convex component to the search space and orienting the search towards a prior estimate $X_{p}$ of the expected solution vector. The regularisation parameter $\alpha \geq 0$ balances the evaluation of individuals between the optimization of the least square criterion, and the agreement with a range of physically admissible solutions. A low value of $\alpha$ implies an insufficient regularisation of the problem, while a high value imposes too much of a constraint and forces the solution to match the prior. Guidelines exist for the correct choice of $\alpha$, such as the L-curve method [15]. This method states that several runs of the search algorithm with different values of $\alpha$ result in an L-shaped graph when displaying the solutions $\left\|X-X_{p}\right\|$ versus their residuals $\left\|Y-Y_{m}\right\|$, and that the optimal choice for $\alpha$ is near the corner of this L-curve. This method is used in the present work to tune the regularisation parameter: the target of the numerical benchmark below (Sec. 3) is to validate the choice of the L-curve method before applying it to an experimental case.

\subsection{Covariance Matrix Adaptation}

The fitness value of an individual $X$ is calculated after the finite-element discretisation of coupled partial differential equations with non-linear transport properties. No simple expression of the fitness function is therefore available, and the Jacobian matrix of the residuals cannot be expressed analytically. It is however possible to estimate this matrix by solving the adjoint system of the problem at each iteration of a Levenberg-Marquardt algorithm [25]. Alternatively, derivative-free metaheuristic methods are appropriate: the present work uses one known as the Covariance Matrix Adaptation Evolution Strategy (CMA-ES) for the resolution of the inverse problem.

The CMA-ES belongs to the category of evolutionary algorithms (EA), along with genetic algorithms and particle swarm optimisation. These algorithms are based on the principle of natural selection to guide the evolution towards a global optimum in a discrete or real-valued search space. A population of individuals is created, evaluated with a pre-defined objective function, and updated by a combination of operators (selection, recombination, mutation) to create the next generation. This process is repeated until some stopping criterion is met. A wide variety of algorithms is made available by the choice of population size, type of selection, crossover operator, mutation probability and strength, the possibility of elitism [10], etc. The term of evolution strategy usually refers to an EA which intrisic properties, or strategy parameters, may vary during the evolution [2].

The principle of CMA-ES is that each generation of $\lambda$ individuals is created following a multivariate normal distribution in $\mathbb{R}^{n}$ whose mean and covariance matrices are adapted after the evaluation of the previous generation [13]. After each generation, the mean of the distribution is moved towards previously successful individuals, while the covariance matrix is adapted as to favor previously successful mutation steps in the future. The selection is of type $(\mu, \lambda)$, in that the $\mu$ best individuals of the parent generation determine the creation of a number $\lambda>\mu$ of offsprings, and no individual from the parent generation is kept unto the next one. This is referred to as a ","selection [2], as opposed to the "+"-selection (or elitism [10]) where each selection process involves both parent and offspring populations.

Although meta-heuristic methods are computationally more expensive than deterministic methods, this drawback is greatly mitigated by the possibility of distributed computing. Indeed, as with other evolutionary algorithms, all function evaluations within a generation are independent: parallelising several calls of the objective function is therefore straightforward. The adaptative mutation strength ensures an initially wide exploration of the search space, while preventing premature convergence and allowing a fine convergence near the optimum [2]. Moreover, the update of the distribution parameters resembles a gradient descent towards a better expected fitness. For these reasons, the CMA-ES is known to perform very well among meta-heuristic methods in real-valued search spaces [12].

In this work, the finite-element code for HAM transfer mentioned above is integrated as the objective function of a CMA algorithm, which is part of the library of evolutionary algorithms $\mathrm{DEAP}^{2}$ [8]. Within each generation,

${ }^{2}$ Distributed Evolutionary Algorithms in Python https://code.google.com/p/deap/ 
separate evaluations of individuals are distributed on several processors using the $\mathrm{SCOOP}^{3}$ module.

\section{A numerical benchmark for calibrating the inverse problem}

\subsection{Setup}

The search algorithm was first tested on a supervised numerical benchmark, as to show its theoretical accuracy before application to real measurements. Data is provided by a preliminary simulation given a choice of material properties, and these properties are then sought by the algorithm. This procedure was already tested in the case of idealised sensors providing noiseless measurements [29], and proved a very good accuracy and repeatability of results, given an appropriate stopping criterion. As actual measurement data is inevitably inaccurate in some extent, the present section extends this study to a slightly more realistic case where reference measurements include noise and a reduced resolution.

We will show that these measurement uncertainties justify the use of some sort of regularisation in acquiring accurate identification results. The second target of the numerical benchmark is to demonstrate this importance, and to show that an existing criterion for the choice of regularisation, the L-curve, is appropriate for our problem. We conducted a parametric study on the regularisation parameter $\alpha$ and aimed at validating the L-curve method for its proper selection.

The setup of the numerical benchmark is depicted by Fig. 1. The physical problem is the coupled heat and moisture transfer through a $10 \mathrm{~cm}$ wood fibre wall during one week. Surface temperature and relative humidity profiles are provided as Dirichlet boundary conditions. Sensors are placed inside the wall: the temperature and relative humidity are "measured" every $10 \mathrm{~min}$ at $x=2.5,5$ and $7.5 \mathrm{~cm}$ and the heat flow is "measured" at $x=5 \mathrm{~cm}$. The procedure is as follows:

1. A reference simulation is run with a known vector of material properties $X^{\star}$. The simulation results are saved at the location of the virtual sensors mentioned above. In the following, these profiles are mentioned as reference data $Y_{m}$.

2. A Gaussian noise is added to all sensor measurements (both boundary conditions and reference data). The standard deviation of the noise is $0.2^{\circ} \mathrm{C}$ on temperature, $1 \%$ on relative humidity and $0.01 \mathrm{~W} / \mathrm{m}^{2}$ on flux measurements. The profiles of interior and exterior boundary conditions are displayed on Fig. 2.

3. The search algorithm attempts to retrieve the material properties on the sole knowledge of the recorded boundary conditions and reference data with noise.

The last step of this list was repeated for several values of the regularisation parameter $\alpha$, each run resulting in a best individual $\widehat{X}_{\alpha}$ regarding the fitness function $f_{\alpha}$. The same prior vector is used for all runs: $X_{p}$ is a set of material properties that have been chosen with errors in the range of $20 \%$ to $25 \%$ regarding the real properties $X^{\star}$. The quality of an estimation is judged on the average error on each component of the vector $\widehat{X}_{\alpha}$ regarding their counterpart in $X^{\star}$ :

$$
e_{\alpha}=\frac{1}{n} \sum_{i=1}^{n} \frac{\left\|\widehat{X}_{\alpha}^{i}-X^{\star, i}\right\|}{X^{\star, i}}
$$

\subsection{Outcome}

The regularisation parameter was tested within a range of $0<\alpha<1000$. The population size was set to $\lambda=12$ and the search was set to stop when the distribution of each parameter $i$ within the population had a low variance $\sigma_{i}$, comparatively to its mean value $\bar{X}_{i}$.

$$
\forall i \in \llbracket 1, n \rrbracket, \sigma_{i} \ll \bar{X}_{i}
$$

The number of objective function calls before convergence decreases with the weight of regularisation: the search stops after 818 generations when $\alpha=10^{-3}$ (9816 calls of $f$ ), and after 69 generations when $\alpha=10^{3}$ (828 calls of $f$ ).

\footnotetext{
${ }^{3}$ Scalable COncurrent Operations in Python http://code.google.com/p/scoop/
} 


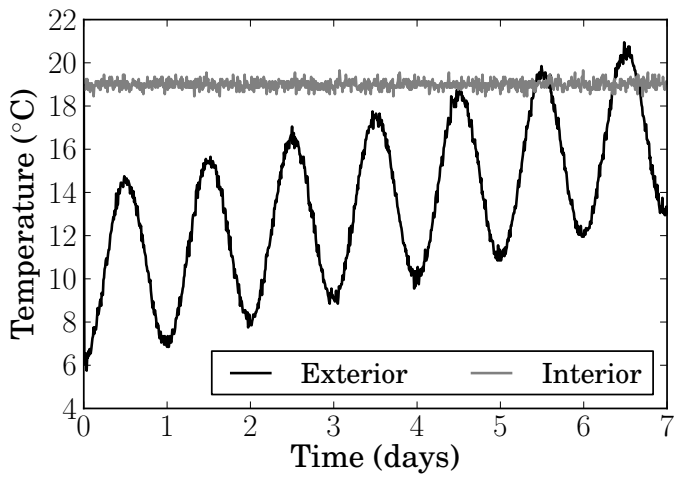

(a)

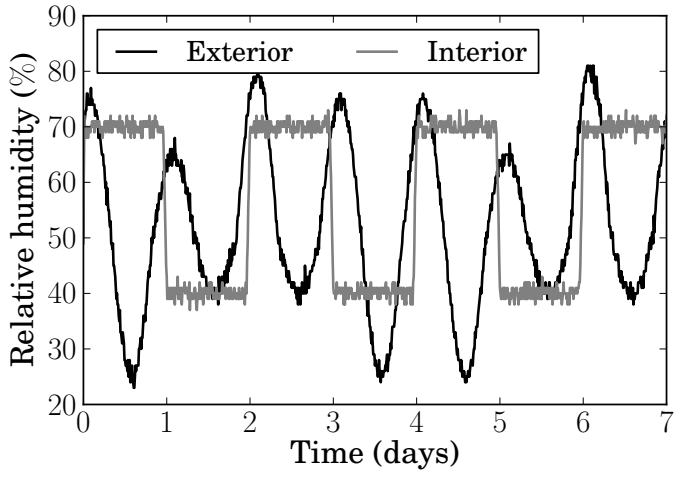

(b)

Figure 2: (a) Temperature and (b) relative humidity boundary conditions of the benchmark with added noise

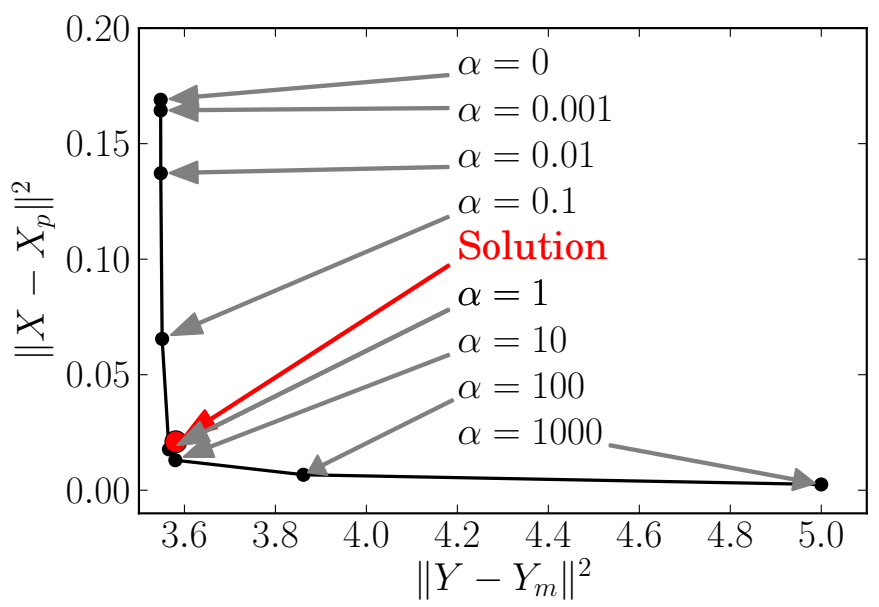

Figure 3: L-curve of the numerical benchmark

This is understandable as regularisation accounts for a smoothing of the search space and filters local optima. As in the preliminary study mentioned above [29], the repeatability has been ensured in the case of $\alpha=0$ by several runs resulting in identical results. The L-curve summarizing the residuals of each search is plotted on Fig. 3.

Each point of the L-curve represents the solution vector $\widehat{X}_{\alpha}$ obtained at the end of a CMA run with one specific value of $\alpha$. The graph must be understood as follows:

- A low $\alpha$ guides the search towards the best possible fit with the experimental data, with little constraint on the value of the parameters in $\mathbb{R}^{n}$. The end result is likely to have physically problematic properties, as the prior information is not well considered.

- A high $\alpha$ guides the search towards the prior and filters measurement data. This results in a higher residual $\left\|Y-Y_{m}\right\|$

- The best compromise is situated at the corner of the L-curve [15]. In an ideal case, this corner is sharp and allows selecting a solution which satisfies both criteria.

In some extent, the L-curve resembles a Pareto front, displaying candidate solutions according to two competing objectives. The choice of $\alpha$ accounts for deciding among candidate solutions in a multi-objective optimisation problem. The difference is that each point is the result of a separate run of the search algorithm. 


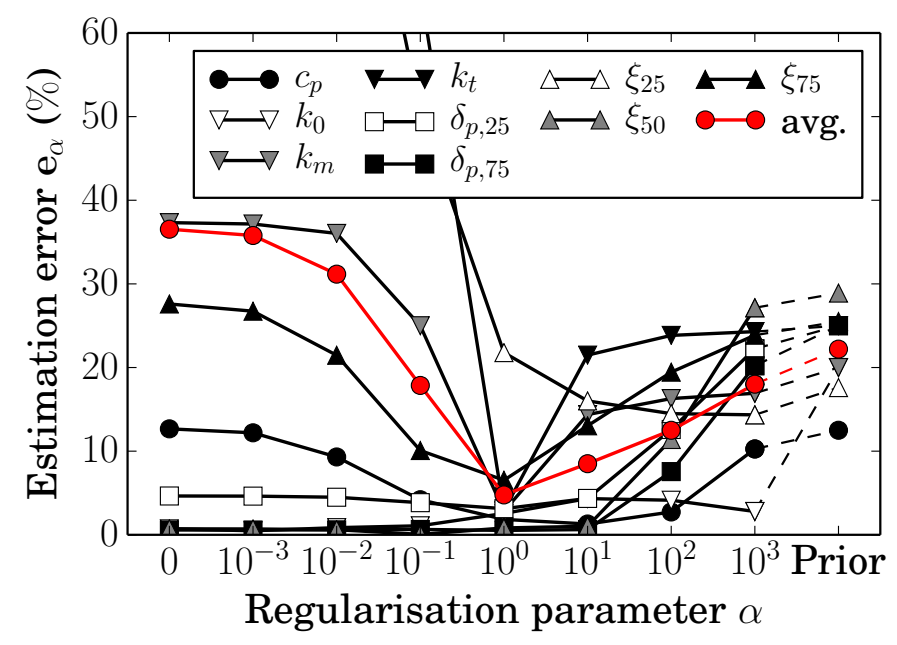

Figure 4: Estimation error of each material property as a function of the regularisation weight

The expected solution of the problem is shown by the red dot on Fig. 3. It is close to the corner of the graph, which is found in the range $1 \leq \alpha \leq 10$. We can now check that candidate solutions $\widehat{X}_{\alpha}$ within this range match the real material properties $X^{\star}$. Fig. 4 displays the estimation error of all properties for each value of $\alpha$.

Interpreting the influence of $\alpha$ can now be done in terms of estimation error:

- No regularisation ( $\alpha \rightarrow 0$, left side of Fig. 4) implies a high identification error. This proves that when measurement data is noisy, the candidate material resulting in the best possible fit is likely to have unrealistic properties. The average error on all parameters is $e_{\alpha=0}=36.5 \%$, with the parameters $k_{t}$ and $\xi_{25 \%}$ exceeding a $100 \%$ deviation from their expected values.

- High regularisation ( $\alpha \rightarrow+\infty$, right side of Fig. 4) makes the solution tend to the prior.

$$
\lim _{\alpha \rightarrow \infty} e_{\alpha}=e_{p}
$$

- The lowest estimation errors for most properties (and the average error) are reached within the range $1 \leq \alpha \leq$ 10. In terms of average error, $e_{\alpha=1}=4.8 \%$ and $e_{\alpha=10}=8.5 \%$.

Numerical results are summarized in Tab. 2. The main outcome of this procedure, is that the range of regularisation parameters resulting in the best estimates of the solution matches the corner of the L-curve. Moreover, it appears that a wide range of values for $\alpha$ yield better results than the prior. Therefore a finer tuning of $\alpha$ does not seem mandatory. The correct choice of $\alpha$ can therefore be done visually on the basis of the L-curve, and it seems that some flexibility is allowed on this parameter.

This numerical benchmark shows that the inverse heat and moisture transfer problem is theoretically achievable, and that a careful formulation of the fitness function may overcome the difficulties rising from sensor inaccuracy. In the best present case $(\alpha=1)$, most parameters are estimated with an error below $3 \%$ and only one with a relatively high error: $\xi_{25 \%}$ with a $21.8 \%$ deviation from its expected value. The temperature dependency of the thermal conductivity, the profile of vapour permeability and even the sorption isotherm are accurately reconstructed.

The analysis of these results can lead to a further investigation. As mentioned earlier, a material property with a high influence on the solution of the forward problem is likely to be easier to identify when solving the inverse problem. One can expect some negative correlation between the sensitivity indices of parameters and their estimation errors. Although a rigorous study of this matter has not been made here, a trend is visible on Fig. 4: influent parameters, such as $c_{p}$ or $k_{0}$, are identified with little error except at very high values of $\alpha$. This is especially true in the case of an insufficient regularisation: low values of $\alpha$ increase the disparity of identification errors between material properties. 
Table 2: Comparison of the exact material properties $X^{\star}$, the prior $X_{p}$, the non-regularised solution $\widehat{X}_{\alpha=0}$ and the selected identification result $\widehat{X}_{\alpha=1}$

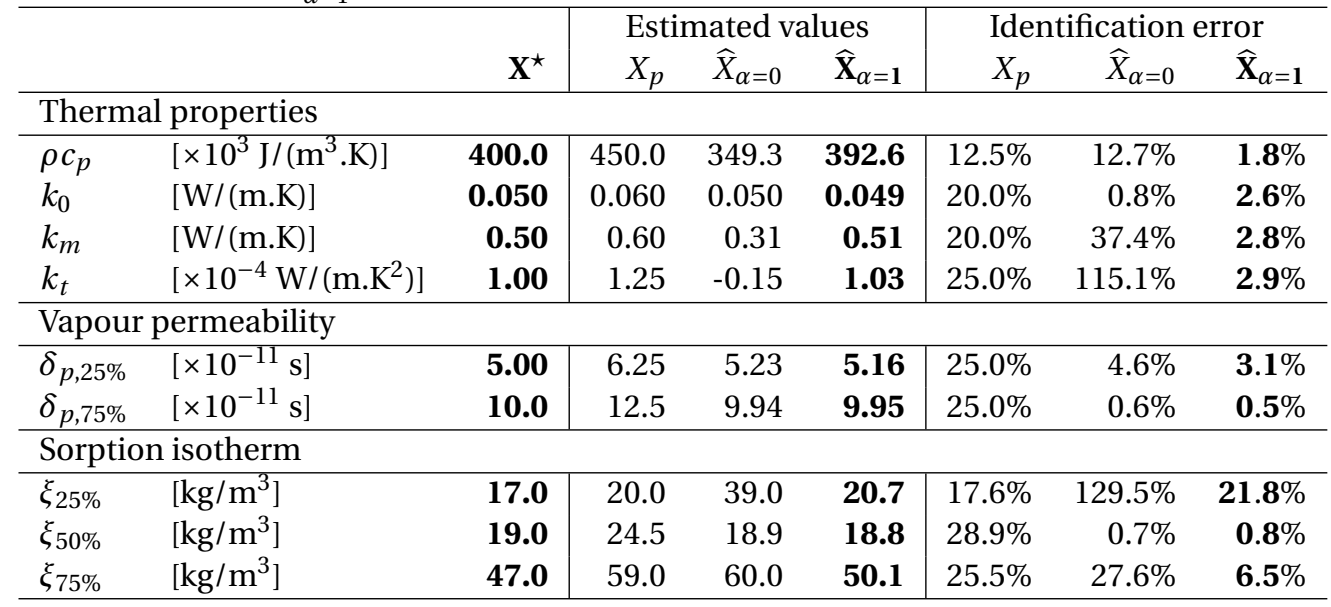

The choice of the CMA-ES algorithm, with an objective function including a term of Tikhonov regularisation, has been validated on a numerical benchmark for the resolution of the inverse heat and moisture transfer problem in building materials. The last step in the present work is to apply this methodology to the real case of an instrumented wall.

\section{Experimental application}

\subsection{Setup}

The experimental validation of the identification procedure is attempted on the basis of measurements previously gathered in the frame of the HYGROBAT project [37]. This project aimed at establishing tools to precisely quantify the impact of mass transfer on heat transfer in highly hygroscopic materials. The CEA-INES institute participated in its experimental part with the PASSYS test cells: controlled conditions are set inside the cells, delimited by an instrumented wall which is exposed to the exterior weather on the other side. Fig. 5 is a picture of a test cell, displaying the instrumented wall on the foreground.

The wall consists of a $16 \mathrm{~cm}$ layer of wood fibre insulation material on the inside, and a $2 \mathrm{~cm}$ coating layer on the outside. Only the first layer is considered in this study. A monitoring period of 2 weeks is used for the model calibration algorithm. The reference data for the fitness evaluation is provided by temperature and humidity sensors located at $x=4,8$ and $12 \mathrm{~cm}$ and by a heat flow sensor located at $x=8 \mathrm{~cm}$ in the wall. The interior $(x=0 \mathrm{~cm})$ and exterior $(x=16 \mathrm{~cm})$ boundary conditions, measured at the material surface, are displayed of Fig. 6 .

The interior boundary conditions are controlled. A constant temperature $T_{i n t}=24.4 \pm 0.6^{\circ} \mathrm{C}$ was maintained. The relative humidity has a set point of $40 \% \mathrm{RH}$ for 1 week, before a step-wise variation to $70 \% \mathrm{RH}$. The exterior boundary conditions are given by the temperature and relative humidity measured at the surface of the wood fibre layer, under a $2 \mathrm{~cm}$ coating layer. There is therefore neither solar radiation nor wind-driven rain directly impacting the investigated material. As in the numerical benchmark above, both boundaries are expressed as Dirichlet conditions in the finite-element simulation of the forward problem.

Separately from the instrumentation of the PASSYS cell, the hygrothermal characterisation of wood fibre was conducted in several institutes involved in the HYGROBAT project [37]. The material properties used below for the validation of the identification are averaged over the results of these participants. 


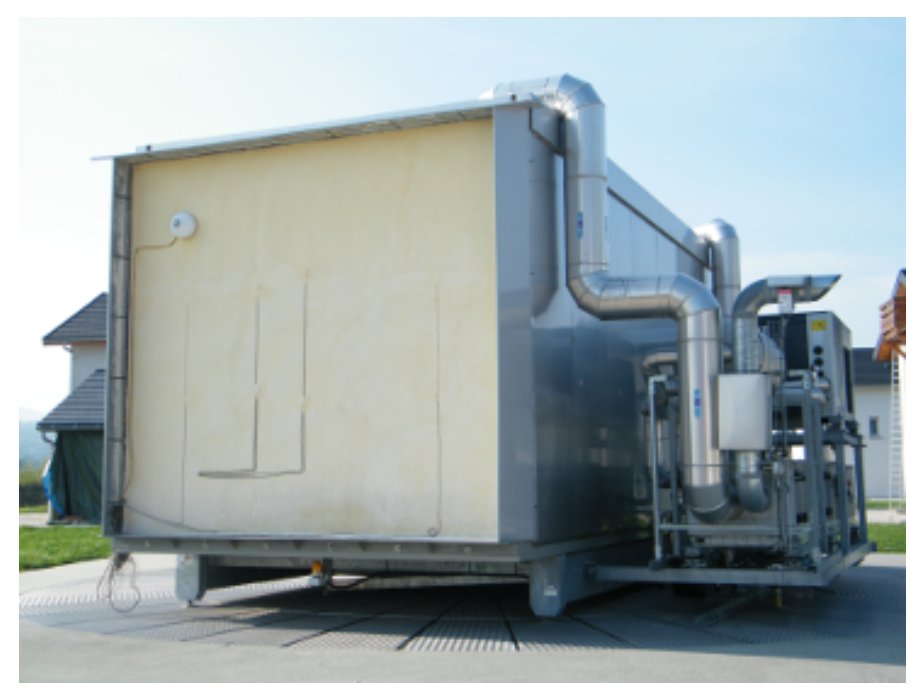

Figure 5: PASSYS instrumented test cell

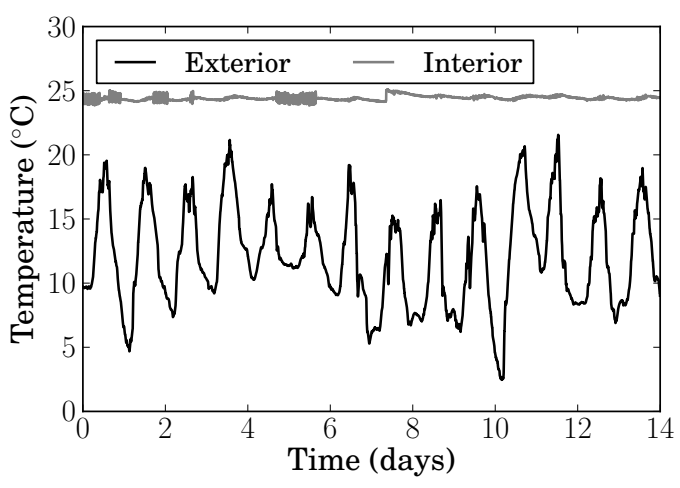

(a)

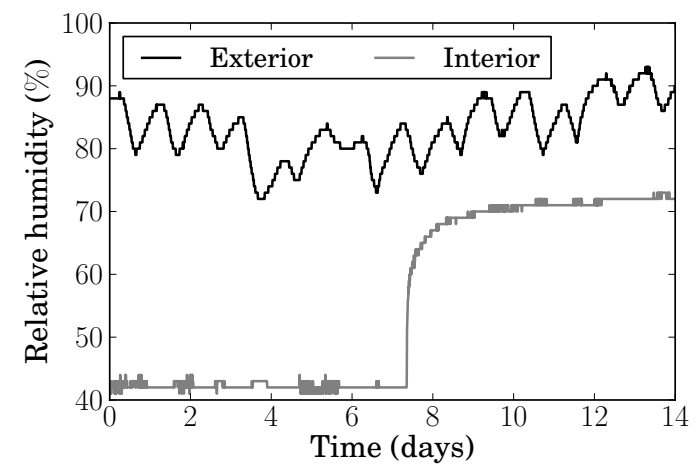

(b)

Figure 6: (a) Temperature and (b) relative humidity boundary conditions of the experimental investigation 


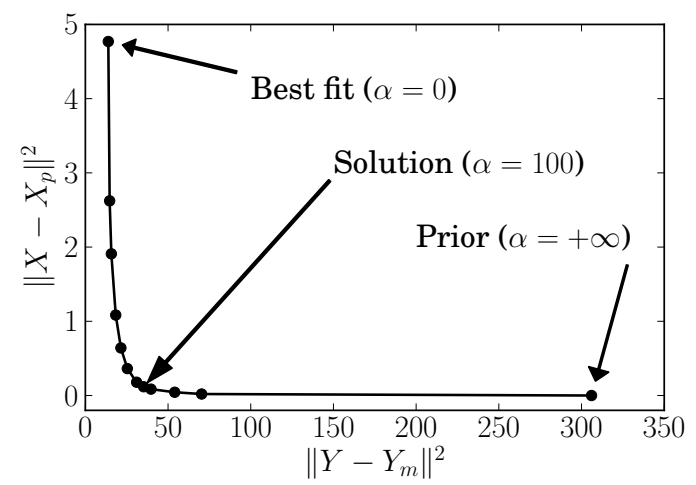

Figure 7: L-curve of the experimental case

\subsection{Outcome}

\subsubsection{Selection of the solution}

The overall procedure for parameter identification is similar from that of the numerical benchmark above: a prior $X_{p}$ is chosen in the range of expected values for the material properties (the precise values of the prior are given in Tab. 4 as a comparison with the identification results and the experimental characterisation). The vector of reference data $Y_{m}$ is provided by the sensors. The search algorithm is run several times, each with a different value of the regularisation parameter $\alpha$ defining the objective function $f_{\alpha}$ (see Eq. 3). The L-curve, Fig. 7, is drawn by showing the residuals of the solution of each run.

As we are now dealing with actual experimental data, the residuals $\left\|Y-Y_{m}\right\|$ and $\left\|X-X_{p}\right\|$ are higher than in the supervised numerical benchmark. The corner of the L-curve is smoother, which does not facilitate the choice for the best compromise. On the basis of this graph, the solution vector $\widehat{X}_{\alpha=100}$ is chosen as the most appropriate solution of the inverse heat and moisture problem. The following discussion will compare several cases in order to assess the quality of this estimation. The terminology of these cases is as shown on Fig. 7:

- "Measured" refers to the experimental data.

- "Best fit" refers to the individual obtained without regularisation $\widehat{X}_{\alpha=0}$, i.e. the best possible numerical fit with the reference data.

- "Prior" refers to simulations run by setting the material properties to those of the prior $X_{p}=\widehat{X}_{\alpha \rightarrow+\infty}$.

- "Solution" refers to the choice $\widehat{X}_{\alpha=100}$ as the best estimated set of parameters.

\subsubsection{Dynamic profiles}

The above specified alternatives are first compared in terms of dynamic temperature, relative humidity and heat flow profiles. Fig. 8 displays the reference data used for model calibration $Y_{m}$ (red lines), the dynamic profiles given by the prior $Y\left(X_{p}\right)$ and the best numerical fit $Y\left(\widehat{X}_{\alpha=0}\right)$ (dotted lines), and the selected solution $Y\left(\widehat{X}_{\alpha=100}\right)$ (black continuous line). On the other hand, the average error between measurements and each of the calculated profiles is summarized in Tab. 3.

One of the requirements for an acceptable solution to the inverse problem is that a fair compromise is made between agreement to the reference data and physical realism of the estimated parameters. Fig. 8 addresses the first concern by showing how the selected set of material properties fits the dynamic measurements. The solution $Y\left(\widehat{X}_{\alpha=100}\right)$ performs generally better than the prior estimation of material properties. This is especially true regarding the humidity and heat flow profiles: HAM simulations using the pre-supposed parameters $X_{p}$ greatly underestimate the kinetics of moisture sorption in the wood fibre layer (see Fig. 8(c) and 8(d)) and overestimate the heat flow in the center of the wall (Fig. 8(e)). On the other hand, the solution $Y\left(\widehat{X}_{\alpha=100}\right)$ has a slightly lower 


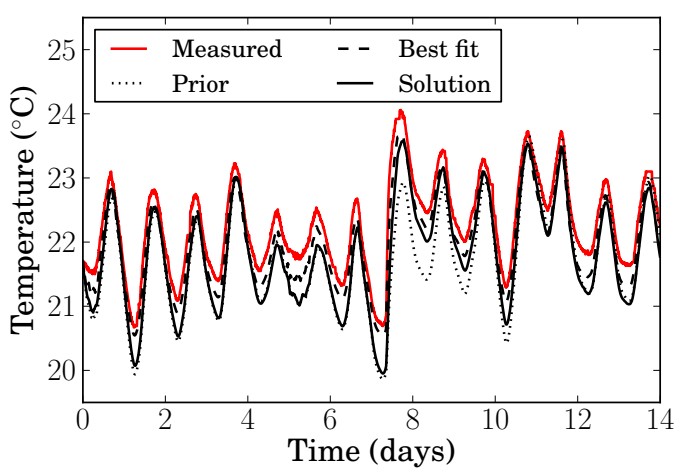

(a) Temperature $(x=4 \mathrm{~cm})$

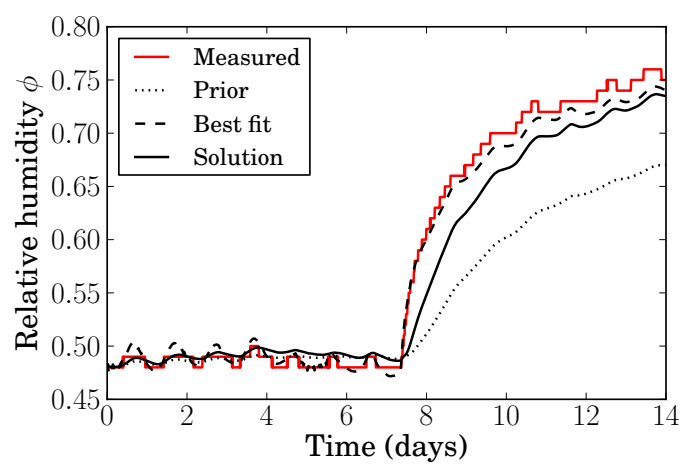

(c) Relative humidity ( $x=4 \mathrm{~cm}$ )

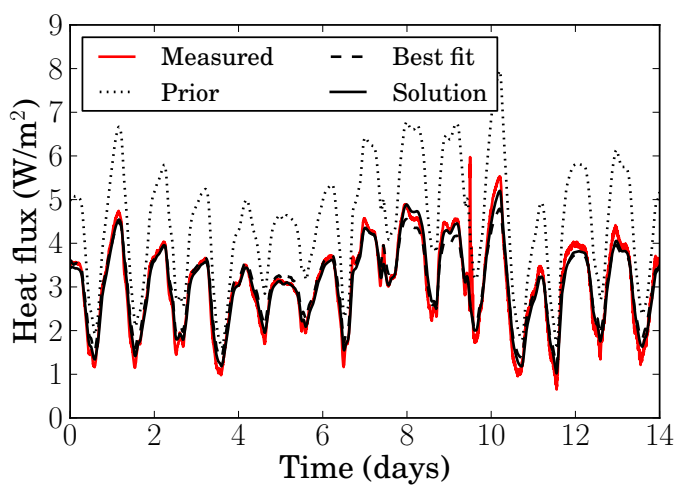

(e) Heat flux $(x=8 \mathrm{~cm})$

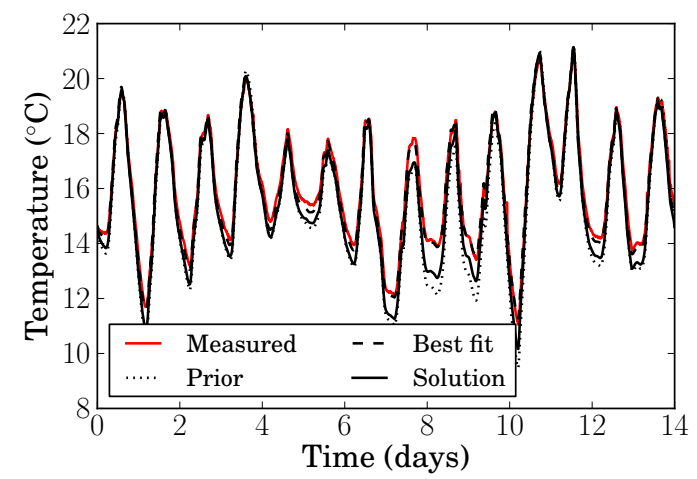

(b) Temperature $(x=12 \mathrm{~cm})$

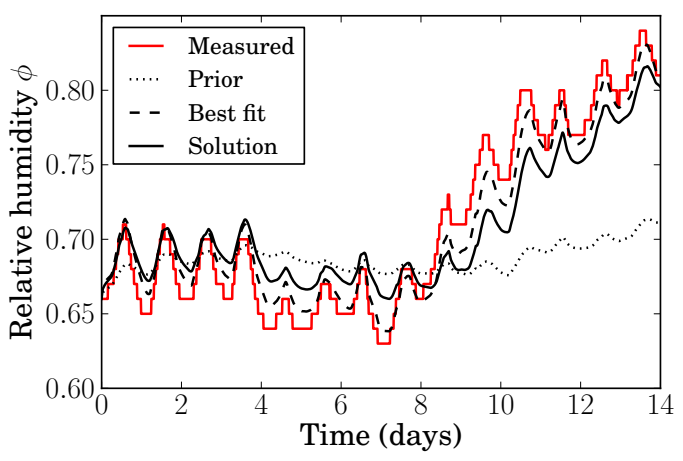

(d) Relative humidity ( $x=12 \mathrm{~cm}$ )

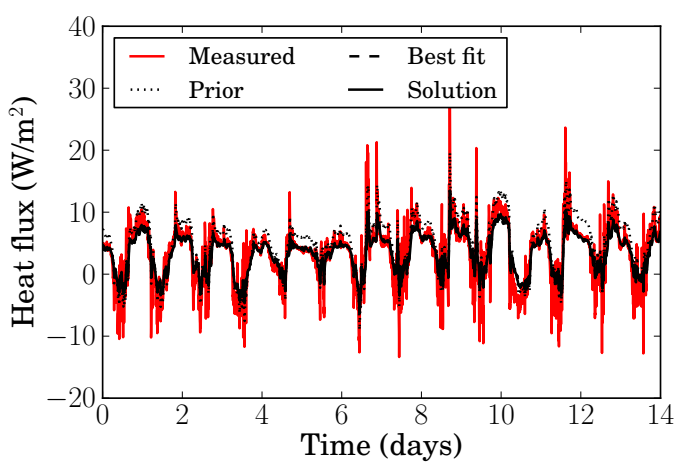

(f) Heat flux ( $x=16 \mathrm{~cm})$

Figure 8: Temperature, relative humidity and heat flux profiles at several locations of the wall

Table 3: Average error on dynamic profiles

\begin{tabular}{lrrr}
\hline & $X_{p}$ & $\widehat{X}_{\alpha=0}$ & $\widehat{\mathbf{X}}_{\alpha=100}$ \\
\hline Temperature & $0.20 \%$ & $0.06 \%$ & $\mathbf{0 . 1 5} \%$ \\
Relative humidity & $6.75 \%$ & $1.22 \%$ & $\mathbf{3 . 0 0} \%$ \\
Heat flow & $49.79 \%$ & $9.15 \%$ & $\mathbf{6 . 5 5} \%$ \\
\hline
\end{tabular}


Table 4: Comparison of the expected material properties $X^{\star}$, the prior $X_{p}$, the non-regularised solution $\widehat{X}_{\alpha=0}$ and the selected identification result $\widehat{X}_{\alpha=100}$

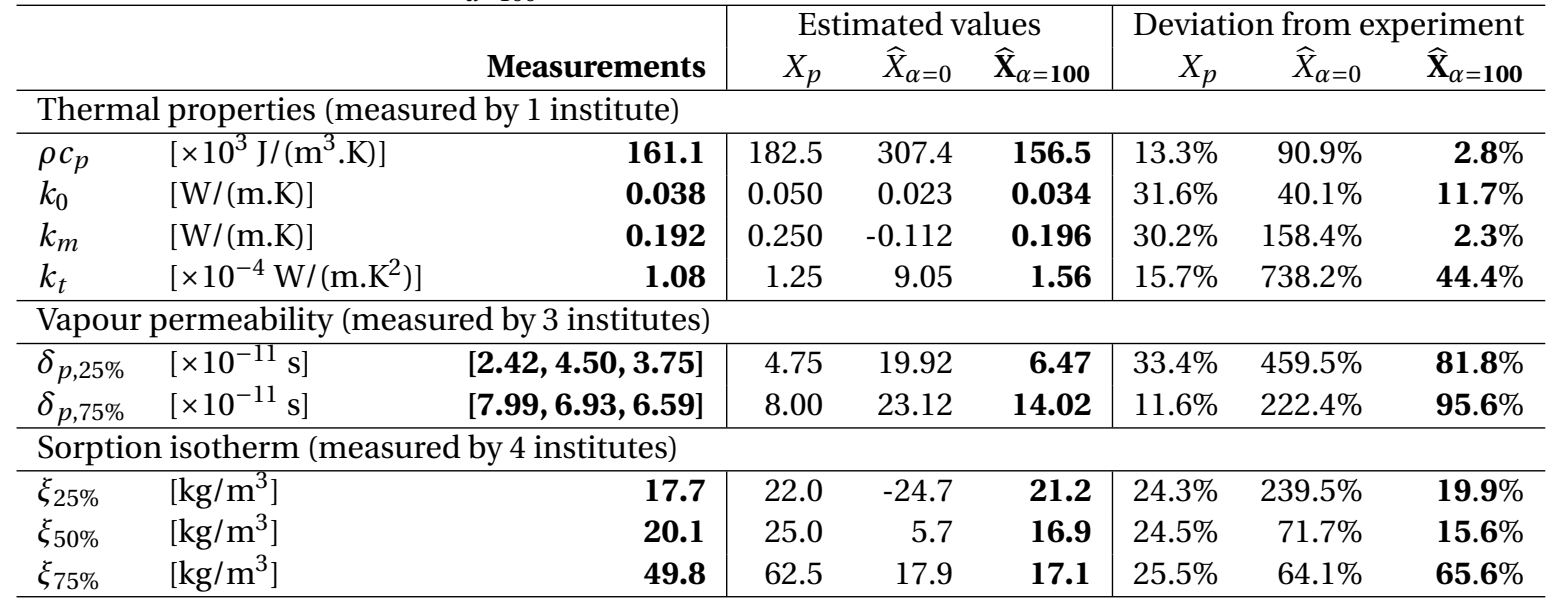

concordance with the experimental data than the best possible fit $Y\left(\widehat{X}_{\alpha=0}\right)$. The average error committed by the solution on the dynamic profiles lies however in a comparable range, as can be seen in Tab. 3 . Note that one of the heat flow profiles (Fig. 8(f)) is not part of the reference data $Y_{m}$ and only serves here as a validation of the simulation results.

\subsubsection{Material properties}

The simulation cases whose results are shown above, are now compared in terms of parameter values. Tab. 4 compares the prior $X_{p}$, the non-regularised solution $\widehat{X}_{\alpha=0}$ and the selected identification result $\widehat{X}_{\alpha=100}$ with the experimental measurements of each property [34]. In the frame of the HYGROBAT project, each material property was measured independently by one or several institutes, as mentioned in the table.

\section{Thermal properties}

Concerning thermal properties $\rho c_{p}$ and $k$, the selected solution $\widehat{X}_{\alpha=100}$ yields a much better agreement with experimental values than both the prior and the non-regularised solution $\widehat{X}_{\alpha=0}$. Only the parameter $k_{t}$ differs significantly from its measured value. This property has however very little influence on the solution of the forward problem. A noteworthy observation is the fact that even properties with a high influence on the forward problem, such as $k_{0}$, are poorly estimated without regularisation.

The moisture-dependent term $k_{m}$ has a limited impact in the present case, because high humidity levels above $90 \%$ were not considered. Tests however show that the thermal conductivity of wood fibre insulation increases several times at high relative humidity when condensation occurs. In this case, $k_{m}$ becomes a more sensitive parameter.

\section{Sorption isotherm}

The sorption isotherm has been experimentally characterised by four participants of the HYGROBAT project, with little deviation between each result. In order to support the discussion regarding this property, the sorption isotherm of each alternative has been reconstructed from the set of parameters $\xi_{i}$ and displayed on Fig. 9.

A first important observation from Tab. 4 and Fig. 9 is how problematic the estimated material properties may become, should the objective function of the inverse problem not include a regularisation term. Indeed, the nonregularised solution $\widehat{X}_{\alpha=0}$, although in a very good match with the dynamic moisture profiles (Fig. 8), has a negative 


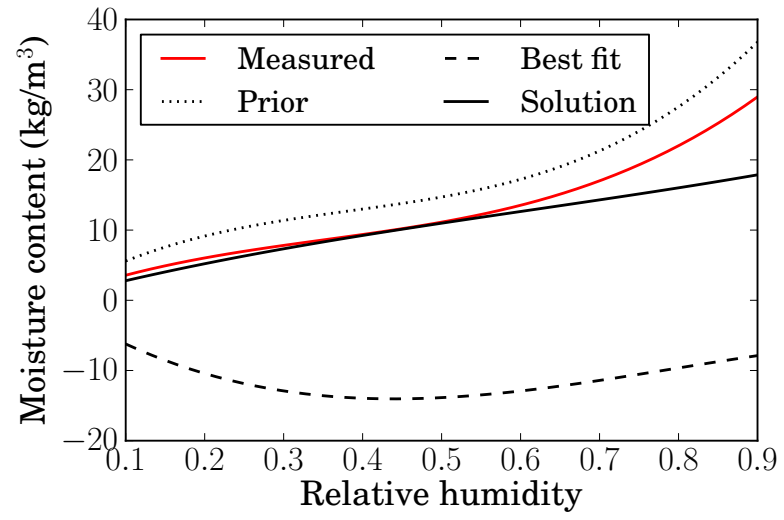

Figure 9: Reconstruction of the sorption isotherm from dynamic measurements

sorption isotherm. Note however that only the slope of the retention curve impacts the simulation results, and that this slope is positive in the humidity levels involved in the present study ( $40 \% \mathrm{RH}$ and higher). The sorption isotherm of the compromise solution $\widehat{X}_{\alpha=100}$ matches the experiment up to $60 \% \mathrm{RH}$, and is underestimated at higher humidity levels.

The mismatch between predicted and measured sorption isotherms is problematic at high RH and may originate from several grounds. One of them is the low parametrisation of the sorption function, described by only three points over the whole humidity range $(25 \%, 50 \%, 75 \%)$. A better precision may be obtainable on the higher slope of the isotherm through a finer parametrisation, although it involves increasing the dimensionality of the inverse problem.

\section{Vapour permeability}

The vapour permeability $\delta_{p}$ has been measured in two ranges of humidity by three participants of the HYGROBAT project, using the dry cup and wet cup methods. The deviation of the calculated permeability with the experimental characterisation is much higher than that of other parameters: the estimated $\delta_{p, 75 \%}$ has twice the value measured by the wet cup experiment. Such a high value is required by the search algorithm, in order to match with the dynamic profiles of relative humidity and minimise the objective function. The dynamics of moisture ingress are indeed greatly underestimated when running the forward problem with the expected value of $\delta_{p}$.

A number of grounds for this disagreement is possible, related to the sources of error of the inverse identification procedure (see Sec. 2.2):

- The parametrisation of the vapour permeability, and its dependency to the relative humidity, may be inappropriate.

- A model hypothesis may be wrong, such as neglecting the presence of liquid water in the material. In this case, the predicted value of $\delta_{p}$ is an equivalent permeability including vapour and liquid transfer effects alike.

- The dry cup and wet cup experiments are stationary measurements, and their results may be inaccurate when applied to the transient simulation of a highly hygroscopic material.

Again, the need for a regularisation parameter in the inverse problem is justified. Indeed, the best fit $Y\left(\widehat{X}_{\alpha=0}\right)$ with the reference data yields an exceedingly high value of the vapour permeability. 
Table 5: Repeatability of the identification with several sensor locations

\begin{tabular}{|c|c|c|c|c|c|c|}
\hline \multicolumn{2}{|c|}{ Sensor locations [cm] } & {$[4 ; 8 ; 12]$} & [4] & [8] & [12] & {$[4 ; 8]$} \\
\hline \multicolumn{2}{|c|}{ Boundary conditions $[\mathrm{cm}]$} & {$[0 ; 16]$} & {$[0 ; 16]$} & {$[0 ; 16]$} & {$[0 ; 16]$} & {$[0 ; 12]$} \\
\hline$\rho c_{p}$ & {$\left[\times 10^{3} \mathrm{~J} /\left(\mathrm{m}^{3} . \mathrm{K}\right)\right]$} & 156.5 & 126.4 & 138.6 & 187.2 & 139.3 \\
\hline$k_{0}$ & [W/(m.K)] & 0.0335 & 0.0335 & 0.0336 & 0.0333 & 0.0365 \\
\hline$k_{m}$ & {$[\mathrm{~W} /(\mathrm{m} \cdot \mathrm{K})]$} & 0.197 & 0.205 & 0.204 & 0.181 & 0.242 \\
\hline$k_{t}$ & {$\left[\times 10^{-4} \mathrm{~W} /\left(\mathrm{m} \cdot \mathrm{K}^{2}\right)\right]$} & 1.56 & 1.48 & 1.54 & 1.65 & 1.28 \\
\hline$\delta_{p, 25 \%}$ & {$\left[\times 10^{-11} \mathrm{~s}\right]$} & 6.47 & 6.05 & 6.50 & 6.55 & 5.61 \\
\hline$\delta_{p, 75 \%}$ & {$\left[\times 10^{-11} \mathrm{~s}\right]$} & 14.0 & 14.3 & 14.2 & 13.9 & 12.1 \\
\hline$\xi_{25 \%}$ & {$\left[\mathrm{~kg} / \mathrm{m}^{3}\right]$} & 21.2 & 20.3 & 21.1 & 22.2 & 21.4 \\
\hline$\xi_{50 \%}$ & {$\left[\mathrm{~kg} / \mathrm{m}^{3}\right]$} & 16.9 & 14.8 & 14.3 & 20.1 & 10.9 \\
\hline$\xi_{75 \%}$ & {$\left[\mathrm{~kg} / \mathrm{m}^{3}\right]$} & 17.1 & 29.8 & 18.6 & 14.8 & 22.4 \\
\hline \multicolumn{2}{|c|}{ Generations before convergenc } & 118 & 110 & 111 & 117 & 113 \\
\hline
\end{tabular}

\subsubsection{Robustness of results}

All estimation results above were obtained with the same number of sensors for recording the reference data $Y_{m}$. An important questioning is whether a large amount of data is necessary for an accurate estimation of material properties. Moreover, the identification procedure can only be considered reliable if its results are reproducible with different sensor locations in a given experimental setup. Thus, the last part of this work is to assess the robustness of the model calibration process by showing if the outcome of the inverse problem depends on the position and number of locations at which data is recorded.

Keeping the same value for the regularisation parameter $\alpha=100$, the search procedure has been repeated with several sets of reference data $Y_{m}$ for the evaluation of candidate material. Three attempts were made, providing the objective function $f$ (Eq. 3) with data from only one sensor, either located at $x=4,8 \mathrm{or} 12 \mathrm{~cm}$. A last attempt was also made with the first two sensors as providers of reference data, while the third one $(x=12 \mathrm{~cm})$ enforced the external boundary condition on the wall. Results of this investigation are shown on Tab. 5 .

On the basis of these results, the repeatability of the identification is satisfactory: when monitoring a wall for the characterisation of its hygrothermal properties, the position of the sensors have little impact on the estimation results (provided that this position is well known).

\section{Conclusion}

The Covariance Matrix Adaptation evolution strategy was used for solving the inverse coupled heat and moisture transfer problem. Provided a set of experimental measurements of temperature, relative humidity and heat flow, this evolutionary algorithm was able to calibrate a HAM model, and give rough estimates of all hygrothermal properties of a building material. The applicability of this procedure was demonstrated in the case of a numerical benchmark, and applied to experimental measurements of a monitored wall. A special focus was made on the importance of including a regularisation term into the fitness function for the evaluation of candidate materials. The L-curve method was applied as a criterion for tuning the regularisation parameter. This method was validated in the case of HAM transfer on the basis of a supervised numerical benchmark. It was shown that a fair choice of regularisation allows overcoming errors arising from measurement noise and model formulation. An experimental application of the procedure was then conducted, by identifying the properties of wood fiber insulation on the basis of a monitored test cell.

Most material properties agree well with their experimental characterisation, especially thermal properties and even in some extent the sorption isotherm. The procedure can be considered very promising for a number of reasons:

- A solution to the inverse problem exists, which yields a good match with dynamic measurements while providing realistic material properties. 
- The performance of the identification algorithm is not strongly dependent from the prior knowledge of the material properties. A rough preliminary estimate of the sought properties is therefore sufficient for an accurate estimation of most parameters.

- The identification results are robust and reproducible when different data sets (several sensor locations) are used. Several outcomes of the identification were compared using one, two or three sensors during the same measurement period. All material properties were estimated using only two weeks of measurements. This is a significant time reduction compared to traditional hygric characterisation techniques. However, there was no attempt at testing different measurement periods, and the study does not indicate how using more or less than two weeks of records would impact the results.

The possibilities for future applications of these results are twofold. First, this methodology can be considered for the development of a new protocol for fast and exhaustive numerically assisted characterisation, improving traditional methods at the laboratory scale. The second possible application is at the building scale: a local estimation of the hygrothermal features of a building envelope allows a better diagnosis in the prospects of building retrofitting. Additional work is necessary before considering in-situ application to multi-layered, heterogeneous walls. The proposed procedure is intrusive as it requires temperature and humidity records within a wall. This work however shows that with an appropriate monitoring equipment, such an in-situ estimation is a realistic prospect.

The question of identification accuracy, and how it is influenced by measurement precision, has been mentioned. Identified properties were compared to experimental measurements, but their confidence intervals have not been rigorously calculated. A local sensitivity study is required to estimate the identification accuracy: the uncertainty on the results of an inverse problem is bounded by the condition number of its sensitivity matrix. A forward sensitivity study would therefore show an estimate of the confidence intervals for the predicted material properties, as functions of the measurement precision. An outlook for this work is to perform such a sensitivity analysis and relate it to measurement precision, since an evolutionary algorithm does not provide this information by itself. Another alternative is the Bayesian approach to inverse problems, which provides complete probability distributions for each inferred parameter. It is an alternative to the evolutionary algorithm used here, which only gives point estimates of the material properties. A future development is the comparison of these two gradient-free inverse methods for building physics applications.

\section{Acknowledgements}

This work uses experimental records that were gathered in the scope of the HYGROBAT project funded by the French National Research Agency (ANR). The authors would like to thank the ANR for supporting this project, which allowed further developments such as presented here.

\section{References}

[1] James V. Beck. Inverse Heat Conduction: Ill-Posed Problems. John Wiley \& Sons, October 1985.

[2] Hans-Georg Beyer and Hans-Paul Schwefel. Evolution strategies - a comprehensive introduction. Natural Computing, 1(1):3-52, March 2002.

[3] Phillip Biddulph, Virginia Gori, Clifford A. Elwell, Cameron Scott, Caroline Rye, Robert Lowe, and Tadj Oreszczyn. Inferring the thermal resistance and effective thermal mass of a wall using frequent temperature and heat flux measurements. Energy and Buildings, 78:10-16, 2014.

[4] George E. P. Box and George C. Tiao. Bayesian Inference in Statistical Analysis. John Wiley \& Sons, January 2011.

[5] Jordan Brouns, Alexandre Nassiopoulos, Frédéric Bourquin, and Karim Limam. State-parameter identification for accurate building energy audits. In Building Simulation 2013 conference, France, August 2013. 
[6] Guy Chavent. Nonlinear Least Squares for Inverse Problems: Theoretical Foundations and Step-by-Step Guide for Applications. Springer, March 2010.

[7] Ralph Evins. A review of computational optimisation methods applied to sustainable building design. Renewable and Sustainable Energy Reviews, 22:230-245, 2013.

[8] Félix-Antoine Fortin, François-Michel De Rainville, Marc-André Gardner, Marc Parizeau, and Christian Gagné. DEAP: Evolutionary algorithms made easy. Journal of Machine Learning Research, 13:2171-2175, July 2012.

[9] D. Gossard, B. Lartigue, and F. Thellier. Multi-objective optimization of a building envelope for thermal performance using genetic algorithms and artificial neural network. Energy and Buildings, 67:253-260, 2013.

[10] Louis Gosselin, Maxime Tye-Gingras, and François Mathieu-Potvin. Review of utilization of genetic algorithms in heat transfer problems. International Journal of Heat and Mass Transfer, 52(9-10):2169-2188, 2009.

[11] Carl-Eric Hagentoft, Angela Sasic Kalagasidis, Bijan Adl-Zarrabi, Staf Roels, Jan Carmeliet, Hugo Hens, John Grunewald, Max Funk, Rachel Becker, Dina Shamir, Olaf Adan, Harold Brocken, Kumar Kumaran, and Reda Djebbar. Assessment method of numerical prediction models for combined heat, air and moisture transfer in building components: Benchmarks for one-dimensional cases. Journal of Thermal Envelope and Building Science, 27(4):327-352, April 2004.

[12] Nikolaus Hansen. The CMA evolution strategy: A comparing review. In Towards a new evolutionary computation. Springer Berlin Heidelberg, 2006.

[13] Nikolaus Hansen and Andreas Ostermeier. Completely derandomized self-adaptation in evolution strategies. Evolutionary Computation, 9(2):159-195, 2001.

[14] P. Hansen. Truncated singular value decomposition solutions to discrete ill-posed problems with illdetermined numerical rank. SIAM Journal on Scientific and Statistical Computing, 11(3):503-518, 1990.

[15] P. Hansen. Analysis of discrete ill-posed problems by means of the l-curve. SIAM Review, 34(4):561-580, 1992.

[16] Y. Heo, R. Choudhary, and G. A. Augenbroe. Calibration of building energy models for retrofit analysis under uncertainty. Energy and Buildings, 47:550-560, 2012.

[17] Hans Janssen, Bert Blocken, and Jan Carmeliet. Conservative modelling of the moisture and heat transfer in building components under atmospheric excitation. International Journal of Heat and Mass Transfer, 50(56):1128-1140, March 2007.

[18] Jari P. Kaipio and Colin Fox. The bayesian framework for inverse problems in heat transfer. Heat Transfer Engineering, 32(9):718-753, 2011.

[19] Jari P. Kaipio and Erkki Somersalo. Statistical and Computational Inverse Problems. Applied Mathematical Science. Springer Verlag, New York, 2005.

[20] Philippe Lauret, Harry Boyer, Carine Riviere, and Alain Bastide. A genetic algorithm applied to the validation of building thermal models. Energy and Buildings, 37(8):858-866, 2005.

[21] Vasileios Machairas, Aris Tsangrassoulis, and Kleo Axarli. Algorithms for optimization of building design: A review. Renewable and Sustainable Energy Reviews, 31:101-112, March 2014.

[22] Laurent Magnier and Fariborz Haghighat. Multiobjective optimization of building design using TRNSYS simulations, genetic algorithm, and artificial neural network. Building and Environment, 45(3):739-746, March 2010. 
[23] Denis Maillet, Yvon Jarny, and Daniel Petit. Problèmes inverses en diffusion thermique : Formulation et résolution du problème des moindres carrés. Techniques de l'ingénieur - Transferts thermiques, base documentaire : TIB214DUO(ref. article : be8266), 2014.

[24] Denis Maillet, Yvon Jarny, and Daniel Petit. Problèmes inverses en diffusion thermique : Outils spécifiques de conduction inverse et de régularisation. Techniques de l'ingénieur - Transferts thermiques, base documentaire : TIB214DUO(ref. article : be8267), 2014.

[25] Alexandre Nassiopoulos and Frédéric Bourquin. On-site building walls characterization. Numerical Heat Transfer, Part A: Applications, 63(3):179-200, 2013.

[26] Anh-Tuan Nguyen, Sigrid Reiter, and Philippe Rigo. A review on simulation-based optimization methods applied to building performance analysis. Applied Energy, 113:1043-1058, January 2014.

[27] Miroslav Raudenský, Keith A. Woodbury, J. Kral, and T. Brezina. Genetic algorithm in solution of inverse heat conduction problems. Numerical Heat Transfer, Part B: Fundamentals, 28(3):293-306, 1995.

[28] Simon Rouchier. Hygrothermal performance assessment of damaged building materials. PhD thesis, Universite Lyon 1, 2012.

[29] Simon Rouchier and Monika Woloszyn. Identification des propriétés hygrothermiques des matériaux par stratégie d'évolution CMA. In IBPSA France 2014 conference, 2014.

[30] Simon Rouchier, Monika Woloszyn, Geneviãilve Foray, and Jean-Jacques Roux. Influence of concrete fracture on the rain infiltration and thermal performance of building facades. International Journal of Heat and Mass Transfer, 61:340-352, 2013.

[31] Andrew M. Stuart. The bayesian approach to inverse problems. arXiv:1302.6989 [math], February 2013.

[32] Andrej Nikolaevich Tikhonov and Vasiliy Yakovlevich Arsenin. Solutions of ill-posed problems. Washington, DC : Winston, 1977.

[33] S. Vakili and M. S. Gadala. Low cost surrogate model based evolutionary optimization solvers for inverse heat conduction problem. International Journal of Heat and Mass Transfer, 56(1-2):263-273, January 2013.

[34] Oly Vololonirina, Marie Coutand, and Bernard Perrin. Vers une méthode de conception HYGRO-thermique des BATiments performants : Propriétés de transferts des matériaux bois. In Colloque International Francophone NoMaD 2012, LMDC-AUGC, 2012.

[35] Jingbo Wang and Nicholas Zabaras. A bayesian inference approach to the inverse heat conduction problem. International Journal of Heat and Mass Transfer, 47(17-18):3927-3941, 2004.

[36] Shengwei Wang and Xinhua Xu. Parameter estimation of internal thermal mass of building dynamic models using genetic algorithm. Energy Conversion and Management, 47(13-14):1927-1941, 2006.

[37] Monika Woloszyn, Nolwenn Le Pierrès, Yannick Kedowidé, Joseph Virgone, Abdelkrim Trabelsi, Zakaria Slimani, and Eric et al. Mougel. Vers une méthode de conception HYGRO-thermique des BATiments performants : démarche du projet HYGRO-BAT. In IBPSA France 2014 conference, 2014.

[38] Xinhua Xu and Shengwei Wang. Optimal simplified thermal models of building envelope based on frequency domain regression using genetic algorithm. Energy and Buildings, 39(5):525-536, 2007.

[39] Chadia Zayane. Identification d'un modèle de comportement thermique de bâtiment à partir de sa courbe de charge. PhD thesis, Ecole Nationale Supérieure des Mines de Paris, January 2011. 